\title{
THREE SOLUTIONS FOR A NONLOCAL PROBLEM WITH CRITICAL GROWTH.
}

\author{
NATALÍ AILÍN CANTIZANO AND ANALÍA SILVA
}

\begin{abstract}
The main goal of this work is to prove the existence of three different solutions (one positive, one negative and one with nonconstant sign) for the equation $\left(-\Delta_{p}\right)^{s} u=|u|^{p_{s}^{*}-2} u+$ $\lambda f(x, u)$ in a bounded domain with Dirichlet condition, where $\left(-\Delta_{p}\right)^{s}$ is the well known $p$ fractional Laplacian and $p_{s}^{*}=\frac{n p}{n-s p}$ is the critical Sobolev exponent for the non local case. The proof follows the ideas of [28] and is based in the extension of the Concentration Compactness Principle for the $p$-fractional Laplacian [20] and Ekeland's variational Principle [7].
\end{abstract}

\section{INTRODUCTION}

Let us consider the following non local equation with Dirchlet boundary conditions

$$
\begin{cases}\left(-\Delta_{p}\right)^{s} u=|u|^{p_{s}^{*}-2} u+\lambda f(x, u) & \text { in } \Omega, \\ u=0 & \text { in } \mathbb{R}^{n} \backslash \Omega .\end{cases}
$$

where $s \in(0,1), \Omega$ is a smooth and bounded domain in $\mathbb{R}^{n}$ and $\left(-\Delta_{p}\right)^{s} u$, called the $p$-fractional Laplacian, is defined up to a normalization constant by

$$
\left(-\Delta_{p}\right)^{s} u:=2 \lim _{\varepsilon \rightarrow 0^{+}} \int_{\mathbb{R}^{n} \backslash B_{\varepsilon}(x)} \frac{|u(x)-u(y)|^{p-2}(u(x)-u(y))}{|x-y|^{n+p s}} d y .
$$

When $p=2$ this is the well known fractional Laplacian. Problems involving non local operators have many applications, just to cite a few, we refer to [6, 8, 13] for some physical models, [1, 16, 23] for some applications in finances, [3] for applications in fluid dynamics, [15, 19, 22] for application in ecology and [14] for some applications in image processing.

The functional framework for this operator are the fractional order Sobolev spaces, see [30] and [5]. The fractional order Sobolev space is defined by

$$
W^{s, p}\left(\mathbb{R}^{n}\right):=\left\{u \in L^{p}\left(\mathbb{R}^{n}\right):[u]_{s, p}<\infty\right\},
$$

where $[u]_{s, p}$ is the famous seminorm of Gagliardo is defined by

$$
[u]_{s, p}:=\left(\int_{\mathbb{R}^{2 n}} \frac{(u(x)-u(y))^{p}}{|x-y|^{n+p s}} d x d y\right)^{\frac{1}{p}},
$$

and $W_{0}^{s, p}(\Omega)$ is defined by $W_{0}^{s, p}(\Omega):=\left\{u \in L^{p}\left(\mathbb{R}^{n}\right):[u]_{s, p}<\infty, u=0\right.$ in $\left.\mathbb{R}^{n} \backslash \Omega\right\}$. It is wellknown that when $s p<n$ the following Sobolev inequality holds

$$
\left(\int_{\mathbb{R}^{n}}|u|^{\frac{n p}{n-s p}} d x\right)^{\frac{n-s p}{n}} \leq C \int_{\mathbb{R}^{2 n}} \frac{|u(x)-u(y)|^{p}}{|x-y|^{n+s p}} d x d y
$$

2010 Mathematics Subject Classification. 35R01,35R11.

Key words and phrases. Sobolev embedding, Non-local, Critical exponents, Concentration compactness. 
for $u \in C_{c}^{\infty}\left(\mathbb{R}^{n}\right)$, where $p_{s}^{*}=\frac{n p}{n-s p}$ is called the critical Sobolev exponent. So, the embedding $W^{s, p}(\Omega) \hookrightarrow L^{q}(\Omega)$ for $1 \leq q \leq p_{s}^{*}$ is continuous. Moreover, is compact for $1 \leq q<p_{s}^{*}$. Critical equations with the fractional Laplacian in bounded domains have been considered in [2, 24, 25, 26, 27]. Multiplicity of solutions for nonlocal equation with critical growth was studied in [11, 21]. The main goal of this paper is to show the existence of three different solutions of the problem (1.1). Moreover these solutions are one positive, one negative and one with non constant sign. We impose adequate conditions on the source $f$ and on the parameter $\lambda$ but we do not impose any parity conditions on the source $f$. This result extends an old paper of Struwe [29]. Similar results for some local operators can be found in [4, 28, 9, 17. The method in the proof used in 29] consists on restricting the functional associated to (1.1) to three different manifolds constructed by imposing a sign restriction and normalizing condition. Then using Ekeland variational principle (see [7]) and a generalization to the fractional setting obtained by Mosconi et al. for any $1<p<\frac{n}{s}$ (see [20]) of the well known Concentration Compactness Principle of P.L.Lions (see [18]), we can prove the existence of a critical point of each restricted functional, that are critical points of the unrestricted one.

Throughout this work, by weak solution of (1.1) we understand critical points of the associated energy functional acting on the Sobolev space $W_{0}^{s, p}(\Omega)$ :

$$
\Phi(u)=\frac{1}{p} \int_{\mathbb{R}^{2 n}} \frac{(u(x)-u(y))^{p}}{|x-y|^{n+p s}} d y d x-\int_{\Omega} \frac{1}{p_{s}^{*}}|u(x)|^{p_{s}^{*}}+\lambda F(x, u(x)) d x,
$$

where $F(x, u)=\int_{0}^{u} f(x, z) d z$.

\section{Assumptions and statement of the Results}

The precise assumptions on the source terms $f$ are as follows:

(H1) $f: \Omega \times \mathbb{R} \rightarrow \mathbb{R}$, is a measurable function with respect to the first argument and continuously differentiable with respect to the second argument for almost every $x \in \Omega$. Moreover, $f(x, 0)=0$ for every $x \in \Omega$.

(H2) There exist constants $c_{1} \in\left(0, \frac{1}{p_{s}^{*}-1}\right), c_{2} \in\left(p, p_{s}^{*}\right), 0<c_{3}<c_{4}$ such that for any $u \in L^{q}(\Omega)$ and $p<q<p_{s}^{*}$,

$$
c_{3}\|u\|_{L^{q}(\Omega)}^{q} \leq c_{2} \int_{\Omega} F(x, u) d x \leq \int_{\Omega} f(x, u) u d x \leq c_{1} \int_{\Omega} f_{u}(x, u) u^{2} d x \leq c_{4}\|u\|_{L^{q}(\Omega)}^{q} .
$$

Remark 2.1. The following example fulfill all of our hypotheses, $f(x, u)=|u|^{q-2} u+\left|u_{+}\right|^{r-2} u_{+}$ if $r \leq q$.

So the main result of the paper reads:

Theorem 2.2. Under the assumptions $(H 1)-(H 2)$, there exist $\lambda^{*}>0$ depending only on $n, p, q$ and the constant $c_{3}$ in (H2), such that for every $\lambda>\lambda^{*}$, there exist three different, nontrivial, (weak) solutions of problem (1.1). Moreover these solutions are, one positive, one negative and the other one has non-constant sing.

\section{Proof of Theorem 2.2}

We will construct three disjoint sets $K_{i}$ not containing 0 such that $\Phi$ has a critical point in $K_{i}$. These sets will be subsets of $C^{1}$-manifolds $M_{i} \subset W_{0}^{s, p}(\Omega)$ that will be constructed by imposing a sing restriction and a normalizing condition. 
In fact,

Definition 3.1. For each $i=1,2,3$, let $M_{i} \subset W_{0}^{s, p}(\Omega)$ be defined as

$$
\begin{gathered}
M_{1}=\left\{u \in W_{0}^{s, p}(\Omega): \int_{\Omega} u_{+}>0 \text { and }\left[u_{+}\right]_{s, p}^{p}-\int_{\Omega}\left|u_{+}\right|^{p_{s}^{*}} d x=\int_{\Omega} \lambda f(x, u) u_{+} d x\right\}, \\
M_{2}=\left\{u \in W_{0}^{s, p}(\Omega): \int_{\Omega} u_{-}>0 \text { and }\left[u_{-}\right]_{s, p}^{p}-\int_{\Omega}\left|u_{-}\right|^{p_{s}^{*}} d x=\int_{\Omega} \lambda f(x, u) u_{-} d x\right\}, \\
M_{3}=M_{1} \cap M_{2},
\end{gathered}
$$

where $u_{+}=\max \{u, 0\}$ and $u_{-}=\max \{-u, 0\}$.

Definition 3.2. For each $i=1,2,3$, let $K_{i} \subset W_{0}^{s, p}(\Omega)$ be defined as

$$
K_{1}=\left\{u \in M_{1}: u \geq 0\right\}, K_{2}=\left\{u \in M_{2}: u \leq 0\right\}, K_{3}=M_{3} .
$$

First, we need the following lemma to show that these sets are nonempty and, moreover, give some properties that will be useful in the proof of our main result.

Lemma 3.3. For every $w_{0} \in W_{0}^{s, p}(\Omega), w_{0}>0\left(w_{0}<0\right)$, there exists $t_{\lambda}>0$ such that $t_{\lambda} w_{0} \in M_{1}$ $\left(\in M_{2}\right)$. Moreover, $\lim _{\lambda \rightarrow \infty} t_{\lambda}=0$.

As a consequence, given $w_{0}, w_{1} \in W_{0}^{s, p}(\Omega), w_{0}>0, w_{1}<0$ with disjoint supports, there exist $\bar{t}_{\lambda}, \underline{t}_{\lambda}$ such that $\bar{t}_{\lambda} w_{0}+\underline{t}_{\lambda} w_{1} \in M_{3}$. Moreover $\bar{t}_{\lambda}, \underline{t}_{\lambda} \rightarrow 0$ as $\lambda \rightarrow \infty$.

Proof. We prove Lemma 3.3 for $M_{1}$, the other cases are analogous.

For $w \in W_{0}^{s, p}(\Omega), w \geq 0$, we consider the functional

$$
\varphi_{1}(w)=[w]_{s, p}^{p}-\int_{\Omega}|w|^{p_{s}^{*}}+\lambda f(x, w) w d x .
$$

Given $w_{0}$, in order to prove the lemma, we must show that $\varphi_{1}\left(t_{\lambda} w_{0}\right)=0$ for some $t_{\lambda}$. Using the hypothesis (H2), we have that:

$$
\varphi_{1}\left(t w_{0}\right) \geq A t^{p}-B t^{p_{s}^{*}}-\lambda c_{4} E t^{q}
$$

and

$$
\varphi_{1}\left(t w_{0}\right) \leq A t^{p}-B t^{p_{s}^{*}}-\lambda c_{3} E t^{q},
$$

where the coefficients $\mathrm{A}, \mathrm{B}$ and $\mathrm{E}$ are given by:

$$
A=\left[w_{0}\right]_{s, p}^{p}, B=\int_{\Omega}\left|w_{0}\right|^{p_{s}^{*}} d x, E=\int_{\Omega}\left|w_{0}\right|^{q} d x .
$$

Since $p<q<p_{s}^{*}$ it follows that $\varphi_{1}\left(t w_{0}\right)$ is positive for a $t$ small enough, and negative for $t$ big enough. Hence, by Bolzano's Theorem, there exists some $t=t_{\lambda}$ such that $\varphi_{1}\left(t_{\lambda} w_{0}\right)=0$.

In order to give an upper bound for $t_{\lambda}$, it is enough to find some $t_{1}$, such that $\varphi_{1}\left(t_{1} w_{0}\right)<0$. We observe that:

$$
\varphi_{1}\left(t w_{0}\right)<A t^{p}-\lambda c_{3} E t^{q}
$$

so it is enough to choose $t_{1}$ such that $A t_{1}^{p}-\lambda c_{3} E t_{1}^{q}=0$, i.e.,

$$
t_{1}=\left(\frac{A}{c_{3} \lambda E}\right)^{\frac{1}{(q-p)}},
$$

therefore, again by Bolzano's Theorem, we can choose $t_{\lambda} \in\left[0, t_{1}\right]$, which implies that $t_{\lambda} \rightarrow 0$ when $\lambda \rightarrow+\infty$, as we wanted to prove. 
For the proof of Theorem 2.2, we need also the following lemmas.

Lemma 3.4. There exist constants $\alpha_{j}>0$ such that, for every $u \in K_{i}, i=1,2,3$,

$$
\alpha_{1}[u]_{s, p}^{p} \leq \alpha_{2}\left(\int_{\Omega}|u|^{p_{s}^{*}}+\lambda f(x, u) u d x\right) \leq \alpha_{3} \Phi(u) \leq \alpha_{4}[u]_{s, p}^{p} .
$$

Proof. As $u \in K_{i}$, we have that

$$
[u]_{s, p}^{p}=\int_{\Omega}|u|^{p_{s}^{*}}+\lambda f(x, u) u d x
$$

choosing $\alpha_{1}=\alpha_{2}$ we have the first inequality.

For the last inequality by (H2)

$$
\int_{\Omega} F(x, u) d x \leq \frac{1}{c_{2}} \int_{\Omega} f(x, u) u d x .
$$

Furthermore,

$$
\left|\lambda \int_{\Omega} F(x, u) d x\right|=\lambda \int_{\Omega} F(x, u) d x \leq \frac{1}{c_{2}} \int_{\Omega} \lambda f(x, u) u d x=\frac{1}{c_{2}}\left([u]_{s, p}^{p}-\int_{\Omega}|u|^{p_{s}^{*}} d x\right),
$$

so

$$
-\lambda \int_{\Omega} F(x, u) d x \leq \frac{1}{c_{2}}\left([u]_{s, p}^{p}-\int_{\Omega}|u|^{p_{s}^{*}} d x\right)
$$

By 3.1, we have:

$$
\begin{aligned}
\Phi(u) & =\frac{1}{p}[u]_{s, p}^{p}-\int_{\Omega} \frac{1}{p_{s}^{*}}|u(x)|^{p_{s}^{*}}+\lambda F(x, u) d x \\
& \leq \frac{1}{p}[u]_{s, p}^{p}-\int_{\Omega} \frac{1}{p_{s}^{*}}|u(x)|^{p_{s}^{*}} d x+\frac{1}{c_{2}}\left([u]_{s, p}^{p}-\int_{\Omega}|u(x)|^{p_{s}^{*}} d x\right) \\
& \leq \frac{1}{p}[u]_{s, p}^{p}+\frac{1}{c_{2}}[u]_{s, p}^{p} \\
& \leq\left(\frac{1}{p}+\frac{1}{c_{2}}\right)[u]_{s, p}^{p} .
\end{aligned}
$$

This proves the third inequality, with $\alpha_{4}=\left(\frac{1}{p}+\frac{1}{c_{2}}\right) \alpha_{3}$.

To prove the middle inequality we proceed as follows:

$$
\begin{aligned}
\Phi(u) & =\frac{1}{p}[u]_{s, p}^{p}-\int_{\Omega} \frac{1}{p_{s}^{*}}|u(x)|^{p_{s}^{*}}+\lambda F(x, u) d x \\
& \geq \frac{1}{p}[u]_{s, p}^{p}-\int_{\Omega} \frac{1}{p_{s}^{*}}|u(x)|^{p_{s}^{*}} d x-\frac{1}{c_{2}} \int_{\Omega} \lambda f(x, u) u d x .
\end{aligned}
$$


So

$$
\begin{aligned}
c_{2} \Phi(u) & \geq c_{2} \frac{1}{p}[u]_{s, p}^{p}-c_{2} \int_{\Omega} \frac{1}{p_{s}^{*}}|u(x)|^{p_{s}^{*}} d x-\int_{\Omega} \lambda f(x, u) u d x \\
& =c_{2} \frac{1}{p}\left(\int_{\Omega}|u(x)|^{p_{s}^{*}} d x+\int_{\Omega} \lambda f(x, u) u d x\right)-c_{2} \int_{\Omega} \frac{1}{p_{s}^{*}}|u(x)|^{p_{s}^{*}} d x-\int_{\Omega} \lambda f(x, u) u d x \\
& =c_{2} \frac{1}{p} \int_{\Omega}|u(x)|^{p_{s}^{*}} d x+c_{2} \frac{1}{p} \int_{\Omega} \lambda f(x, u) u d x-c_{2} \int_{\Omega} \frac{1}{p_{s}^{*}}|u(x)|^{p_{s}^{*}} d x-\int_{\Omega} \lambda f(x, u) u d x \\
& =c_{2}\left(\frac{1}{p}-\frac{1}{p_{s}^{*}}\right) \int_{\Omega}|u(x)|^{p_{s}^{*}} d x+\left(c_{2} \frac{1}{p}-1\right) \int_{\Omega} \lambda f(x, u) u d x .
\end{aligned}
$$

Since $\gamma_{1}=c_{2}\left(\frac{1}{p}-\frac{1}{p_{s}^{*}}\right)$ and $\gamma_{2}=\left(c_{2} \frac{1}{p}-1\right)$ are positive, we take $\alpha_{2}=\min \left\{\gamma_{1}, \gamma_{2}\right\}, \alpha_{3}=c_{2}$ and we have

This finishes the proof.

$$
\alpha_{3} \Phi(u) \geq \alpha_{2}\left(\int_{\Omega}|u|^{p_{s}^{*}}+\lambda f(x, u) u d x\right) .
$$

Lemma 3.5. There exists a constant $D$ such that $\left[u_{+}\right]_{s}^{p} \geq D$, for all $u \in K_{1},\left[u_{-}\right]_{s, p}^{p} \geq D$ for all $u \in K_{2}$, and $\left[u_{-}\right]_{s, p}^{p},\left[u_{+}\right]_{s}^{p} \geq D$ for all $u \in K_{3}$.

Proof. By definition of $K_{i}$ we have

$$
\left[u_{ \pm}\right]_{s, p}^{p}=\left\|u_{ \pm}\right\|_{p_{s}^{*}}^{p_{*}^{*}}+\int_{\Omega} \lambda f(x, u) u_{ \pm} d x .
$$

Using (H2) we have

$$
\int_{\Omega} \lambda f(x, u) u_{ \pm} d x \leq c_{4}\left\|u_{ \pm}\right\|_{q}^{q}, \text { for } p_{s}^{*} \geq q>p
$$

Then

$$
\left[u_{ \pm}\right]_{s, p}^{p} \leq\left\|u_{ \pm}\right\|_{p_{s}^{*}}^{p^{*}}+c_{4}\left\|u_{ \pm}\right\|_{q}^{q} \leq \tilde{C}\left(\left[u_{ \pm}\right]_{s}^{p_{s}^{*}}+\left[u_{ \pm}\right]_{s}^{q}\right)
$$

In the second inequality we use Poincaré inequality. In summary $\left[u_{+}\right]_{s, p}^{p} \leq \hat{C}\left[u_{ \pm}\right]_{s, p}^{r}$. Where $r=q$ if $\left[u_{ \pm}\right]_{s, p}<1$ or $r=p_{s}^{*}$ if $\left[u_{ \pm}\right]_{s, p} \geq 1$. Since $r>p$ we have what we need.

The following lemma describes the properties of the manifolds $M_{i}$.

Lemma 3.6. $M_{i}$ is a sub-manifold of $W_{0}^{s, p}(\Omega)$ of codimension 1 , if $i=1,2$ and 2 if $i=3$ respectively, the sets $K_{i}$ are complete, and for every $u \in M_{i}$ we have $T_{u} W_{0}^{s, p}(\Omega)=T_{u} M_{i} \oplus$ $\operatorname{span}\left\{u_{+}, u_{-}\right\}$where $T_{u} M$ is the tangent space at $u$ of the Banach manifold $M$. Finally, the projection to the first coordinate is uniformly continuous on $M_{i}$.

Proof. We consider

$$
\begin{gathered}
\overline{M_{1}}=\left\{u \in W_{0}^{s, p}(\Omega): \int_{\Omega} u_{+}>0\right\}, \\
\overline{M_{2}}=\left\{u \in W_{0}^{s, p}(\Omega): \int_{\Omega} u_{-}>0\right\}, \\
\overline{M_{3}}=\overline{M_{1}} \cap \overline{M_{2}} .
\end{gathered}
$$

Observe that $M_{i} \subset \overline{M_{i}}$ and since the sets $\overline{M_{i}}$ are open so it's sufficient to prove that $M_{i}$ is a regular sub-manifold of $W_{0}^{s, p}(\Omega)$. 
We are going to build a function $C^{1}, \varphi: \overline{M_{i}} \rightarrow \mathbb{R}^{d}$ with $d=1$ if $i=1,2$ or $d=2$ if $i=3$, such that $M_{i}$ is the inverse of a regular value of $\varphi_{i}$.

We define

$$
\begin{aligned}
& \varphi_{1}(u)=\left[u_{+}\right]_{s, p}^{p}-\int_{\Omega}\left|u_{+}\right|^{p_{s}^{*}}+\lambda f(x, u) u_{+} d x \quad \text { for } u \in M_{1}, \\
& \varphi_{2}(u)=\left[u_{-}\right]_{s, p}^{p}-\int_{\Omega}\left|u_{-}\right|^{p_{s}^{*}}+\lambda f(x, u) u_{-} d x \quad \text { for } u \in M_{2},
\end{aligned}
$$

and

$$
\varphi_{3}(u)=\left(\varphi_{1}(u), \varphi_{2}(u)\right) \text { for } u \in M_{3} .
$$

We have that $M_{i}=\varphi_{i}^{-1}(0)$ so we have to prove that 0 is a regular value of $\varphi_{i}$.

Let us calculate $\left\langle\nabla \varphi_{1}(u), u_{+}\right\rangle$for $u \in M_{1}$,

$$
\frac{d}{d \varepsilon} \varphi_{1}\left(u+\varepsilon u_{+}\right)=\frac{d}{d \varepsilon}\left(\left[\left(u+\varepsilon u_{+}\right)_{+}\right]_{s, p}^{p}-\int_{\Omega}\left|\left(u+\varepsilon u_{+}\right)_{+}\right|^{p_{s}^{*}}+\lambda f\left(x, u+\varepsilon u_{+}\right)\left(u+\varepsilon u_{+}\right)_{+} d x\right) .
$$

Since $\left(u+\varepsilon u_{+}\right)_{+}=u_{+}+\varepsilon u_{+}$we have that $\frac{d}{d \varepsilon} \varphi_{1}\left(u+\varepsilon u_{+}\right)$is equal to

$$
(1+\varepsilon)^{p-1} p\left[u_{+}\right]_{s, p}^{p}-\int_{\Omega} p_{s}^{*}(1+\varepsilon)^{p_{s}^{*}-1}\left|u_{+}\right|^{p_{s}^{*}}+\lambda f\left(x, u+\varepsilon u_{+}\right) u_{+}+\lambda f_{u}\left(x, u+\varepsilon u_{+}\right)(1+\varepsilon) u_{+}^{2} d x,
$$

then since $u \in M_{1}$,

$$
\begin{aligned}
\left.\frac{d}{d \varepsilon} \varphi_{1}\left(u+\varepsilon u_{+}\right)\right|_{\varepsilon=0} & =\left(p\left[u_{+}\right]_{s, p}^{p}-\int_{\Omega} p_{s}^{*}\left|u_{+}\right|^{p_{s}^{*}}+\lambda f(x, u) u_{+}+\lambda f_{u}(x, u) u_{+}^{2} d x\right) \\
& \leq p_{s}^{*}\left(\left[u_{+}\right]_{s, p}^{p}-\int_{\Omega}\left|u_{+}\right|^{p_{s}^{*}} d x\right)-\int_{\Omega} \lambda f(x, u) u_{+}+\lambda f_{u}(x, u) u_{+}^{2} d x \\
& =p_{s}^{*}\left(\int_{\Omega} \lambda f(x, u) u_{+} d x\right)-\int_{\Omega} \lambda f(x, u) u_{+}+\lambda f_{u}(x, u) u_{+}^{2} d x \\
& =\left(p_{s}^{*}-1\right)\left(\int_{\Omega} \lambda f(x, u) u_{+} d x\right)-\int_{\Omega} \lambda f_{u}(x, u) u_{+}^{2} d x .
\end{aligned}
$$

By $\left(H_{2}\right)$ we know that there exists $c_{1} \in\left(0, \frac{1}{p_{s}^{*}-1}\right)$ such that

$$
\int_{\Omega} \lambda f(x, u) u_{+} d x \leq c_{1} \int_{\Omega} \lambda f_{u}(x, u) u_{+}^{2} d x
$$

Then

$$
\left(p_{s}^{*}-1\right) \int_{\Omega} \lambda f(x, u) u_{+} d x-\int_{\Omega} \lambda f_{u}(x, u) u_{+}^{2} d x<0 .
$$

In summary, we have that $\left\langle\nabla \varphi_{1}(u), u_{+}\right\rangle<0$, then $\nabla \varphi_{1}(u) \neq 0$. This means that $M_{1}$ is a regular submanifold of $W_{0}^{s, p}(\Omega)$.

The proof for $M_{2}$, is analogous.

Let's observe that if we prove that $\left\langle\nabla \varphi_{2}(u), u_{+}\right\rangle=\left\langle\nabla \varphi_{1}(u), u_{-}\right\rangle=0$ for $u \in M_{3}$ then for what we had made before, we know that $\left\langle\nabla \varphi_{1}(u), u\right\rangle<0$ and $\left\langle\nabla \varphi_{2}(u), u\right\rangle<0$. For this we can affirm that $\nabla \varphi_{3}(u) \neq 0$ for $u \in M_{3}$. 
Then we will prove that $\left\langle\nabla \varphi_{1}(u), u_{-}\right\rangle=0$. In fact,

$$
\begin{aligned}
\frac{d}{d \varepsilon} \varphi_{1}\left(u+\varepsilon u_{-}\right) & =\frac{d}{d \varepsilon}\left(\left[\left(u+\varepsilon u_{-}\right)_{+}\right]_{s, p}^{p}-\int_{\Omega}\left|\left(u+\varepsilon u_{-}\right)_{+}\right|^{p_{s}^{*}}+\lambda f\left(x, u+\varepsilon u_{-}\right)\left(u+\varepsilon u_{-}\right)_{+} d x\right) \\
& =\frac{d}{d \varepsilon}\left(\left[u_{+}\right]_{s, p}^{p}-\int_{\Omega}\left|u_{+}\right|^{p_{s}^{*}}+\lambda f\left(x, u+\varepsilon u_{-}\right) u_{+} d x\right) \\
& =-\int_{\Omega} \lambda f_{u}\left(x, u+\varepsilon u_{-}\right) u_{+} u_{-} d x=0 .
\end{aligned}
$$

Then

$$
\left.\frac{d}{d \varepsilon} \varphi_{1}\left(u+\varepsilon u_{-}\right)\right|_{\varepsilon=0}=0 .
$$

In an analogous way we have $\left\langle\nabla \varphi_{2}(u), u_{+}\right\rangle=0$. Therefore, $M_{3}$ is a regular submanifold.

The completeness of $K_{i}$ is easy and is left to the reader.

Finally, it remains to see that

$$
T_{u} W_{0}^{s, p}(\Omega)=T_{u} M_{1} \oplus \operatorname{span}\left\{u_{+}\right\},
$$

where $M_{1}=\left\{u: \varphi_{1}(u)=0\right\}$ and $T_{u} M_{1}=\left\{v:\left\langle\nabla \varphi_{1}(u), v\right\rangle=0\right\}$. Now let $v \in T_{u} W_{0}^{s, p}(\Omega)$ be a unit tangential vector, then $v=v_{1}+v_{2}$ where $v_{2}=\alpha u_{+}$and $v_{1}=v-v_{2}$. Let us take $\alpha$ as

$$
\alpha=\frac{\left\langle\nabla \varphi_{1}(u), v\right\rangle}{\left\langle\nabla \varphi_{1}(u), u_{+}\right\rangle} .
$$

With this choice, we have that $v_{1} \in T_{u} M_{1}$. Now

$$
\left\langle\nabla \varphi_{1}(u), v_{1}\right\rangle=0 .
$$

The very same argument is used to show that $T_{u} W_{0}^{s, p}(\Omega)=T_{u} M_{2} \oplus \operatorname{span}\left\{u_{-}\right\}$and $T_{u} W_{0}^{s, p}(\Omega)=$ $T_{u} M_{i} \oplus \operatorname{span}\left\{u_{+}, u_{-}\right\}$.

From these formulas and the estimates given in the first part of the proof, the uniform continuity of the projections onto $T_{u} M_{i}$ follows.

Now, we say that $\left\{u_{j}\right\} \subset W_{0}^{s, p}(\Omega)$ is a Palais-Smale sequence of $c$ level if

(i) $\Phi\left(u_{j}\right) \rightarrow c$,

(ii) $\nabla \Phi\left(u_{j}\right) \rightarrow 0$ in $W^{-s, p}(\Omega)$.

We say that $\Phi$ satisfies Palais-Smale condition of level $c$ if for every $\left\{u_{j}\right\}$ Palais-Smale sequence of level $c$ there exists a subsequence that converges strongly in $W_{0}^{s, p}(\Omega)$.

Now, in order to use Ekeland's variational principle, we need to check the Palais-Smale condition for the functional $\Phi$ restricted to the manifold $M_{i}$. To this end, we need the following lemma which proves the Palais-Smale condition for the unrestricted functional below certain energy level.

Lemma 3.7. The unrestricted functional $\Phi$ verifies the Palais-Smale condition for energy level c for every $c<\frac{s}{n} S^{\frac{n}{s p}}$, where $S$ is the best Sobolev constant for the fractional Laplacian $S:=$ $\inf _{\phi \in C_{c}^{\infty}(\Omega)} \frac{[\phi]_{s, p}^{p}}{\|\phi\|_{p_{s}^{*}}^{p}}$.

The proof of Lemma 3.7 is omitted as it uses standard ideas and is based in the Concentration Compactness Principle for nonlocal operators (see[20]). For the local case it can be found in [12, 28]. For the non local case it follows similarly, see [10] for the details. 
Now, we can prove the Palais-Smale condition for the restricted functional.

Lemma 3.8. The functional $\left.\Phi\right|_{K_{i}}$ satisfies the Palais-Smale condition for energy level $c$ for every $c<\frac{s}{n} S^{\frac{n}{s p}}$.

Proof. Let $\left\{u_{k}\right\} \subset K_{i}$ be a Palais-Smale sequence, that is $\Phi\left(u_{k}\right)$ is uniformly bounded and $\left.\nabla \Phi\right|_{K_{i}} \rightarrow 0$ strongly. We need to show that there exists a subsequence $u_{k_{j}}$ that converges strongly in $K_{i}$.

Let $v_{j} \in T_{u_{j}} W_{0}^{s, p}(\Omega)$ be a unit tangential vector such that

$$
\left\langle\nabla \Phi\left(u_{j}\right), v_{j}\right\rangle=\left\|\nabla \Phi\left(u_{j}\right)\right\|_{W_{0}^{-s, p}(\Omega)} \cdot
$$

Now, by lemma 3.6, $v_{j}=w_{j}+z_{j}$ with $w_{j} \in T_{u_{j}} M_{i}$ and $z_{j} \in \operatorname{span}\left\{\left(u_{j}\right)_{+},\left(u_{j}\right)_{-}\right\}$.

Since $\Phi\left(u_{j}\right)$ is uniformly bounded, by Lemma 3.4 $u_{j}$ is uniformly bounded in $W_{0}^{s, p}(\Omega)$ and hence $w_{j}$ is uniformly bounded in $W_{0}^{s, p}(\Omega)$. Therefore

$$
\left\|\nabla \Phi\left(u_{j}\right)\right\|_{W_{0}^{-s, p}(\Omega)}=\left\langle\nabla \Phi\left(u_{j}\right), v_{j}\right\rangle=\left\langle\left.\nabla \Phi\right|_{K_{i}}\left(u_{j}\right), v_{j}\right\rangle \rightarrow 0 .
$$

As $v_{j}$ is uniformly bounded and $\left.\nabla \Phi\right|_{K_{i}}\left(u_{j}\right) \rightarrow 0$ strongly, the inequality converges strongly to 0 . Now the result follows by Lemma 3.7

We now immediately obtain the following lemma.

Lemma 3.9. There exists $u \in K_{i}$ be a critical point of the restricted functional $\left.\Phi\right|_{K_{i}}$. Moreover $u$ is also a critical point of the unrestricted functional $\Phi$ and hence a weak solution to (1.1).

With all this preparatives, this is the proof of our main result.

Proof of Theorem 2.2. To prove the Theorem [2.2, we need to check that the functional $\left.\Phi\right|_{K_{i}}$ verifies the hypotheses of the Ekeland's Variational Principle.

The fact that $\Phi$ is bounded below over $K_{i}$ is a direct consequence of the construction of the manifold $K_{i}$.

Then by Ekeland's Variational Principle, there exists $v_{k} \in K_{i}$, such that

$$
\Phi\left(v_{k}\right) \rightarrow c_{i} \text { and }\left(\left.\nabla \Phi\right|_{K_{i}}\right)\left(v_{k}\right) \rightarrow 0 .
$$

We have to check that if we choose $\lambda$ large, we have that $c_{i}<\frac{s}{n} S^{\frac{n}{s p}}$. This follows easily from Lemma 3.3. For instance, for $c_{1}$ we have that choosing $w_{0} \geq 0$,

$$
c_{1} \leq \Phi\left(t_{\lambda} w_{0}\right) \leq \frac{1}{p} t_{\lambda}^{p}\left[w_{0}\right]_{s, p}^{p} .
$$

Moreover, it follows from the estimate of $t_{\lambda}$ in Lemma 3.3, that $c_{1} \rightarrow 0$ as $\lambda \rightarrow 0$. Then $c_{i}<\frac{s}{n} S^{\frac{n}{s p}}$ for $\lambda>\lambda^{*}\left(p, q, n, c_{3}\right)$. The other cases are analogous.

From Lemma 3.7, it follows that $v_{k}$ has a convergent subsequence, that we still call $v_{k}$. Therefore $\Phi$ has a critical point in $K_{i}, i=1,2,3$ and, by construction, one of them is positive, other is negative and the last one changes sign. 


\section{ACKNOWLEDGEMENTS}

We want to thank Julián Fernández Bonder for his valuable help.

This paper was supported by grants UBACyT 20020130100283BA, CONICET PIP 11220150100032CO and PROICO 031906, UNSL.

N.Cantizano is a fellow of CONICET and A.Silva is member of CONICET.

\section{REFERENCES}

[1] Vedat Akgiray and G. Geoffrey Booth. The siable-law model of stock returns. Journal of Business $6 \mathcal{G}$ Economic Statistics, 6(1):51-57, 1988.

[2] B. Barrios, E. Colorado, A. de Pablo, and U. Sánchez. On some critical problems for the fractional Laplacian operator. J. Differential Equations, 252(11):6133-6162, 2012.

[3] Peter Constantin. Euler equations, Navier-Stokes equations and turbulence. In Mathematical foundation of turbulent viscous flows, volume 1871 of Lecture Notes in Math., pages 1-43. Springer, Berlin, 2006.

[4] Pablo L. De Nápoli, Julián Fernández Bonder, and Analía Silva. Multiple solutions for the $p$-Laplace operator with critical growth. Nonlinear Anal., 71(12):6283-6289, 2009.

[5] Eleonora Di Nezza, Giampiero Palatucci, and Enrico Valdinoci. Hitchhiker's guide to the fractional Sobolev spaces. Bull. Sci. Math., 136(5):521-573, 2012.

[6] Qiang Du, Max Gunzburger, R. B. Lehoucq, and Kun Zhou. Analysis and approximation of nonlocal diffusion problems with volume constraints. SIAM Rev., 54(4):667-696, 2012.

[7] I. Ekeland. On the variational principle. J. Math. Anal. Appl., 47:324-353, 1974.

[8] A. Cemal Eringen. Nonlocal continuum field theories. Springer-Verlag, New York, 2002.

[9] Julián Fernández Bonder. Multiple positive solutions for quasilinear elliptic problems with sign-changing nonlinearities. Abstr. Appl. Anal., 2004(12):1047-1055, 2004.

[10] Julián Fernández Bonder, Nicolas Saintier, and Analía Silva. The concentration-compactness principle for fractional order sobolev spaces in unbounded domains and applications to the generalized fractional brezisnirenberg problem. https://arxiv.org/abs/1802.09322.

[11] Alessio Fiscella, Giovanni Molica Bisci, and Raffaella Servadei. Multiplicity results for fractional Laplace problems with critical growth. Manuscripta Math., 155(3-4):369-388, 2018.

[12] J. García Azorero and I. Peral Alonso. Multiplicity of solutions for elliptic problems with critical exponent or with a nonsymmetric term. Trans. Amer. Math. Soc., 323(2):877-895, 1991.

[13] Giambattista Giacomin and Joel L. Lebowitz. Phase segregation dynamics in particle systems with long range interactions. I. Macroscopic limits. J. Statist. Phys., 87(1-2):37-61, 1997.

[14] Guy Gilboa and Stanley Osher. Nonlocal operators with applications to image processing. Multiscale Model. Simul., 7(3):1005-1028, 2008.

[15] Nicolas et al. Humphries. Environmental context explains lévy and brownian movement patterns of marine predators. Nature, 465:1066-1069, 2010.

[16] Sergei Levendorski. Pricing of the american put under lévy processes. International Journal of Theoretical and Applied Finance, 7(03):303-335, 2004.

[17] Yuanyuan Li. The existence of solutions for quasilinear elliptic problems with multiple Hardy terms. Appl. Math. Lett., 81:7-13, 2018.

[18] P.-L. Lions. The concentration-compactness principle in the calculus of variations. The limit case. I. Rev. Mat. Iberoamericana, 1(1):145-201, 1985.

[19] Annalisa Massaccesi and Enrico Valdinoci. Is a nonlocal diffusion strategy convenient for biological populations in competition? J. Math. Biol., 74(1-2):113-147, 2017.

[20] Sunra Mosconi and Marco Squassina. Nonlocal problems at nearly critical growth. Nonlinear Anal., 136:84101, 2016.

[21] Kanishka Perera, Marco Squassina, and Yang Yang. Bifurcation and multiplicity results for critical fractional p-Laplacian problems. Math. Nachr., 289(2-3):332-342, 2016.

[22] A. M. Reynolds and C. J. Rhodes. The lévy flight paradigm: random search patterns and mechanisms. Ecology, 90(4):877-887, 2009.

[23] Wim Schoutens. Lévy Processes in Finance: Pricing Financial Derivatives. Willey Series in Probability and Statistics. Willey, New York, 2003. 
[24] Raffaella Servadei. The Yamabe equation in a non-local setting. Adv. Nonlinear Anal., 2(3):235-270, 2013.

[25] Raffaella Servadei. A critical fractional Laplace equation in the resonant case. Topol. Methods Nonlinear Anal., 43(1):251-267, 2014.

[26] Raffaella Servadei and Enrico Valdinoci. A Brezis-Nirenberg result for non-local critical equations in low dimension. Commun. Pure Appl. Anal., 12(6):2445-2464, 2013.

[27] Raffaella Servadei and Enrico Valdinoci. Fractional Laplacian equations with critical Sobolev exponent. Rev. Mat. Complut., 28(3):655-676, 2015.

[28] Analia Silva. Multiple solutions for the $p(x)$-Laplace operator with critical growth. Adv. Nonlinear Stud., 11(1):63-75, 2011.

[29] Michael Struwe. Three nontrivial solutions of anticoercive boundary value problems for the pseudo-Laplace operator. J. Reine Angew. Math., 325:68-74, 1981.

[30] Hans Triebel. Theory of function spaces. Modern Birkhäuser Classics. Birkhäuser/Springer Basel AG, Basel, 2010. Reprint of 1983 edition [MR0730762], Also published in 1983 by Birkhäuser Verlag [MR0781540].

A. Silva

Instituto de Matemática Aplicada San Luis, imasl.

Universidad Nacional de San Luis and CONICET.

EJERCito DE LOS ANDES 950.

D5700HHW San Luis, Argentina.

E-mail address: asilva@dm.uba.ar

URL, A.Silva: https://analiasilva.weebly.com/

N. Cantizano

Instituto de Matemática Aplicada San Luis, IMASL.

Universidad Nacional de San Luis and CONICET.

EJERCito DE LOS ANDES 950.

D5700HHW San Luis, Argentina.

E-mail address: ncantizano@unsl.edu.ar 